\title{
Variables que influyen sobre el aprendizaje del inglés como segunda lengua
}

\author{
Josefina del Carmen Santana Villegas \\ Universidad Panamericana \\ jsantana@up.edu.mx \\ Arturo García-Santillán \\ UCC Business School / Universidad Cristóbal Colón. Campus Calasanz \\ arturogarciasantillan@yahoo.com.mx \\ Milka Elena Escalera-Chávez \\ Universidad Autónoma de San Luis Potosí SLP-México \\ milkaech@uaslp.mx.
}

Resumen: El aprendizaje del idioma inglés cobra cada vez mayor auge en el mundo, sin embargo la brecha entre la calidad del aprendizaje de una segunda lengua entre los estudiantes es cada vez mayor. Por ello el objetivo de esta investigación es explorar las variables que puedan llevar a un mejor aprendizaje del inglés. El estudio es de tipo cuantitativo, la muestra está integrada por 897 alumnos de nuevo ingreso de una universidad privada. Se aplicó un análisis de Regresión Lineal Múltiple y un análisis ANOVA, los resultados muestran las variables: tiempo de estudio, tipo de escuela, y la frecuencia con qué se lee influyen en un mayor puntaje en el examen de ubicación de inglés; además se observó una diferencia significativa entre las variables que miden la percepción del alumno sobre sus habilidades para este idioma y el puntaje obtenido.

Palabras clave: inglés como segunda lengua; adquisición de una lengua extranjera; variables. 
Abstract: Learning English is becoming increasingly important around the world. However, the gap in the quality of language studies between different types of institutions is also growing. Thus, this study aims to explore the variables which may lead to better learning of the English language. The study is quantitative; the sample is 897 students entering a private university. A Multiple Linear Regression and an ANOVA were applied. The results show that the variables: hours of instruction, type of school, and frequency of reading in the second language impact on a greater score in an English language placement test. A significant difference was observed among variables which measure students' perception of their language abilities and the test score.

Keywords: English as a Second Language; acquisition of a foreign language; variables.

\section{Introducción}

El aprendizaje del idioma inglés cobra cada vez mayor auge en el mundo. El inglés es el idioma de la tecnología, del comercio y de las ciencias (Graddol, 2006), por lo que hablantes de otras lenguas en diversos países ven en el aprendizaje del inglés la llave de ingreso a estudios, empleos, y mayores ingresos. Esto ha sido la tendencia en América Latina, en donde México, Colombia, Chile, Brasil, Argentina, Ecuador, Uruguay, Honduras, Perú, Costa Rica, y Paraguay han implementado políticas públicas encaminadas a promover la enseñanza del inglés en sus respectivos países (Sánchez \& Diez, 2014).

De los países antes mencionados, México, fue el primero en implementar las políticas que incluyeran el inglés de manera obligatoria en la escuela secundaria y en el bachillerato. Esto fue en 1993. En la década pasada, la Secretaría de Educación Pública (SEP) de México se propuso incluir el inglés desde la educación básica. El Programa Nacional de Inglés en Educación Básica (PNIEB) ha significado un esfuerzo importante del gobierno, aun cuando no haya rendido los frutos esperados por diversos motivos. En cuanto a las instituciones de educación superior, la SEP ha señalado como objetivo «Alentar la enseñanza de, al menos, una segunda lengua (principalmente el inglés) como parte de los planes de estudios, y propiciar su inclusión como requisito de egreso de la educación superior» (SEP, 2007; p. 45).

Un estudio reciente realizado por la iniciativa Mexicanos Primero, señala lo poco efectivo que han sido estos esfuerzos al sentenciar que «en México, el inglés se ha enseñado poco, mal y tarde» (O’Donoghue \& Calderón, 2015; p. 73). El 
estudio de Sánchez y Diez (2014) señala que México obtuvo un índice medio de calificación en dominio de la lengua. El resto de la región no está mejor.

Este es el panorama en cuanto al inglés en la educación pública en México. En el sector privado, las cosas no marchan tan mal. El acceso a más horas de clase en grupos más pequeños y con docentes más preparados puede contribuir a que, en las escuelas privadas, los alumnos sí logren un mejor dominio del idioma inglés. Davies (2009) menciona un estudio comparativo entre los niveles de inglés alcanzados por egresados de institutos de educación superior del sector público y el sector privado en México. El estudio encontró una fuerte correlación entre el nivel socio-económico- con sus consiguientes ventajas- y el nivel de inglés obtenido en el examen. En la universidad pública mencionada en el estudio, el 7\% de los alumnos mostró un nivel superior de inglés; el 78\% mostró un nivel básico o elemental. En cambio, en la universidad privada, sólo el 16\% se ubicó en nivel básico o elemental, y el $41 \%$ alcanzó un nivel superior.

El presente estudio tiene como objetivo explorar las variables que puedan llevar a un mejor aprendizaje del inglés en la educación básica, media, y media superior. Se correlaciona el nivel de inglés alcanzado en un examen de ubicación realizado al ingreso a una universidad privada, con algunas variables tales como tiempo de estudio, tipo de institución, y tipo de docente.

Son pocos los estudios que se han abocado a entender el porqué de la brecha entre la calidad del aprendizaje de una segunda lengua en los sectores educativos públicos y privados; los pocos que existen tienden a ser cualitativos (Mejía, 2016). Entender cuáles son las variables que realmente inciden en un aprendizaje efectivo será necesario a fin de utilizar de manera más eficiente los recursos disponibles y lograr el bilingüismo nacional buscado.

\section{Estado de la cuestión}

La adquisición de una segunda lengua ha sido objeto de investigación durante varias décadas. La mayoría de los lingüistas cree en la hipótesis de la diferencia fundamental en la adquisición de una segunda lengua, que señala que el aprendizaje de una segunda lengua obedece a un proceso distinto al aprendizaje de la primera lengua- la lengua materna- sobre todo en adultos. Los adultos tienden a ser más analíticos, por lo que aprovechan mejor la enseñanza directa y explícita de conceptos lingüísticos. (Fromkin, Rodman \& Hyams, 2011; Brown, 2000). El dominio de la propia lengua también afecta el dominio de la segunda lengua (Lightbown \& Spada, 2013). Este hecho también coadyuva a que los adultos sean mejores aprendices que los niños. 
Aunque casi todo mundo aprende sin dificultad su primera lengua, no es así para la segunda lengua. La facilidad o la dificultad para aprender una segunda lengua dependen de variables tanto individuales como sociales. Entre las diferencias individuales podemos dividir las características en dos grupos: lo cognitivo y lo afectivo (Brown, 2000; Lightbown \& Spada, 2013; Mitchell \& Myles, 2004). El contexto social en el cual se da el aprendizaje también es un factor de éxito o fracaso en el aprendizaje. Este incluye elementos tales como las oportunidades que se tienen para aprender, ya sea de manera formal (en el aula) o informal (fuera del aula, en un país donde se habla el idioma de manera nativa) (Mitchell \& Myles, 2004).

\subsection{Factores cognitivos}

Los factores cognitivos incluyen la inteligencia, la aptitud, y el uso de estrategias de aprendizaje. Se ha señalado, por ejemplo, que quienes demuestran una inteligencia mayor al promedio tienden a aprender mejor una segunda lengua, sobre todo si este aprendizaje se da dentro del contexto formal de un aula (Mitchell $\&$ Myles, 2004).

Aunque es difícil distinguir la aptitud para un idioma de la inteligencia en general, algunos estudios, (por ejemplo, Gardner \& MacIntyre, 1992, citados en Mitchell \& Myles, 2004) demuestran que algunas habilidades específicas se correlacionan con la facilidad para aprender un idioma. Estas son: habilidad para la codificación fonética, sensibilidad gramatical, memoria, aprendizaje inductivo.

De acuerdo con Harley \& Hart (1997) citados en Mitchell y Myles (2004) estas habilidades han demostrado ser los mejores predictores de éxito en el aprendizaje de una segunda lengua.

Algunos estudios — por ejemplo el de Ranta (2002), o el de Erard (2012), ambos citados en Lightbown \& Spada (2013) — han mostrado que alumnos con facilidad para analizar el lenguaje tienden a ser más exitosos en el aprendizaje, incluyendo quienes estudian en programas sin enfoque gramatical: «... los aprendices que tienen mayor aptitud son capaces de descifrar las reglas del idioma basados en el input» (p. 19). Asimismo, los aprendices exitosos muestran, además de aptitud, una disposición para trabajar duro para lograr sus metas.

El uso de estrategias de aprendizaje es otro de los factores cognitivos. Aunque se ha demostrado que los aprendices más capaces tienden a utilizar más las estrategias de aprendizaje, no es claro si son mejores aprendices porque utilizan las estrategias, o si utilizan estrategias porque son buenos aprendices. Las estrategias que mayor impacto tienen sobre el aprendizaje de la segunda lengua son las metacognitivas (Brown, 2000; Santana, 2005). 


\subsection{Factores afectivos}

El campo afectivo incluye una variedad de factores: empatía, autoestima, extraversión, inhibición, imitación, ansiedad, actitudes, entre otras. Las actitudes que se tienen hacia la lengua son de particular importancia ya que, cuanto mayor sea el interés del aprendiz en el idioma y su cultura, más fácil será su aprendizaje.

La actitud está muy ligada a la motivación. Gardner \& MacIntyre (1992) citados en Mitchell and Myles (2004) entienden motivación como el deseo de lograr una meta, el esfuerzo realizado para alcanzar dicha meta, y la satisfacción obtenida por lograr la meta. Diversos estudios han mostrado una significativa correlación entre la motivación por aprender un idioma y su consiguiente logro.

Un último factor afectivo decisivo es el de la ansiedad ante los idiomas, un fenómeno que ha sido muy estudiado y documentado (Horwitz, 1988, entre otros). Este impacta de manera negativa en el desempeño. Su contraparte, la autoconfianza, o la disposición para comunicarse (Willingness to Communicate o WTC), contribuyen a lograr el dominio de la segunda lengua (Lightbown \& Spada, 2013). Esto se debe al importante papel desempeñado por el output- la posibilidad de poner en práctica lo aprendido, ya sea en un ambiente formal, como el aula, o en un ambiente informal, como es la vida cotidiana.

La importancia del input -acceso al ejemplo del lenguaje hablado y escrito-es ampliamente reconocida. Sin embargo, es poco lo que se ha escrito de la importancia del output- el uso estructurado del lenguaje. Swain (1985) y sus colegas (Swain \& Lapkin, 1995) destacan que no se necesita conocer la gramática de un idioma para entenderlo, pero sí es necesario para la creación de mensajes. Por ello, el output puede ser más efectivo aún que el input para el logro del dominio del idioma (Mitchell \& Myles, 2004).

Es necesario también considerar el contexto sociocultural en el aprendizaje de la segunda lengua. El aprendizaje no sólo se da dentro de un entorno social, sino que la razón de ser del aprendizaje es social: uno aprende una segunda lengua a fin de comunicarse con otros.

\subsection{La edad como factor en el aprendizaje}

Contrario a lo que comúnmente se cree, la edad en que se comienza a estudiar no parece ser un factor determinante en el aprendizaje de una segunda lengua. Un estudio realizado por Lightbown (2012) citado en Lightbown \& Spada (2013), demostró que la edad no es tan importante como el número de horas de instrucción recibida. Muñoz (2006) menciona un estudio en el que comparó el desempeño de aprendices españoles que habían comenzado a estudiar el inglés a los 8 , 
11, o 14 años, o después de los 18. En cada medición, los aprendices tardíos se desempeñaron mejor que quienes iniciaron sus estudios a los 8 años.

Por su parte, Brown (2000) señala que los niños de siete años o menos no tienen mayor ventaja en el aprendizaje de un segundo idioma que los niños de 11 o 12. Existen fuertes evidencias de que existe un período crítico para la adquisición del acento, pero únicamente de eso. Cuanto más pequeño inicia el niño sus estudios, mejor será su acento; quienes retrasan el aprendizaje hasta después de la adolescencia tendrán siempre un «acento extranjero». En México, y en algunos otros países también, hay una fuerte tendencia a querer implementar el estudio del inglés desde la escuela pre-escolar. Será importante cuestionar dicha tendencia si la única ventaja consiste en lograr un acento mejor.

A medida que los gobiernos se plantean implementar la enseñanza del idioma inglés, se incrementan los estudios que pretenden indagar sobre la efectividad de los programas oficiales. continuación se detallan algunos estudios recientes que pueden aportar a la presente investigación.

Ardasheva y Tretter (2013) realizaron un estudio entre 840 estudiantes no nativos de inglés quienes cursaban desde el tercer hasta el décimo año en 37 escuelas en Estados Unidos. Se utilizó el Modelo jerárquico lineal para la investigación, mostrando que cuatro variables- el dominio del idioma oral, las estrategias metacognitivas, la habilidad para leer en el idioma nativo, y la calidad de enseñanza en la escuela- contribuyen de manera importante a la comprensión lectora en el segundo idioma

Un estudio realizado en Chile (Rodríguez, 2013) utilizando análisis inferencial bivariante buscó saber los niveles de logro diferenciados entre los egresados de escuelas públicas y los de escuelas particulares. Se usaron para este fin las bases de datos nacionales, que incluyen información sobre más de 65 mil alumnos. El estudio demostró que los niveles de logro en inglés son muy bajos, tanto en comprensión lectora como auditiva en ambas situaciones educativas, con una leve ventaja para los colegios privados. El mejor aprovechamiento alcanzado por estos se explica básicamente por las características socioeconómicas del alumnos. Una vez controlada esta variable, la mejor capacidad en competencias comunicativas se da en los centros educativos públicos.

Baker-Smemoe, Dewey, Bown \& Martinsen (2014) estudiaron 102 parlantes de inglés quienes participaron en programas de intercambio a varios países: México, España, Francia, Rusia, Egipto y China para entender los factores que entran en juego para que se aproveche o no la estancia en el extranjero para aprender el idioma local. Las variables estudiadas fueron: tiempo en el extranjero, oportunidades para usar el idioma, sensibilidad cultural, sexo y edad de los participantes, 
personalidad, y redes sociales del participante (el tamaño, la dispersión y la densidad de las redes). Encontraron que las variables que mejor predicen el incremento en competencia fueron la sensibilidad cultural y las redes sociales.

Un estudio español, (Valero \& Jiménez, 2015) examinó la posible existencia de una dificultad específica para aprender una segunda lengua. Se entrevistaron a docentes y tutores especializados para detectar alumnos con bajo rendimiento, y entender si la dificultad se daba sólo en el ámbito de aprendizaje del inglés, o si se podía generalizar a otros ámbitos. El estudio mostró que $79 \%$ de los alumnos que no aprueban la materia muestra problemas generalizados. Sólo el 21\% presenta una dificultad específica para el aprendizaje del inglés como segunda lengua.

Por otra parte, en un estudio colombiano (Mejía, 2016), se buscó explicar el porqué de la brecha de nivel de inglés entre colegios públicos y privados, y si esta se ha reducido a raíz de la implementación de política gubernamentales encaminadas a alcanzar el bilingüismo en ese país. Se compararon los resultados de los exámenes nacionales de inglés aplicados en 2008 y en 2013. No se encontraron cambios importantes en cuanto al desempeño, pero sí se encontró un incremento en la motivación para estudiar el idioma.

Por último, un estudio realizado en Costa Rica (López, et al., s/f) habla del considerable impacto del contexto social sobre el aprendizaje de una segunda lengua, debido, en parte, al acceso que tienen los estudiantes de niveles socio-económicos más altos al internet, los libros, las obras de arte y otros bienes culturales.

\section{Diseño y Método}

El estudio presentado se caracteriza por ser no experimental de corte transversal, que se aborda desde el paradigma positivista cuantitativo, el cual busca explicar cuál o cuáles variables como: Vivir en el extranjero, Razones para estudiar el idioma, Frecuencia de lectura en inglés, Años de estudio, Tipo de escuela, Clases particulares y Clases en algún instituto.

\subsection{Participantes}

Los participantes fueron alumnos de nuevo ingreso en la Universidad Panamericana - una universidad privada del occidente de México- quienes tomaban un examen de ubicación de inglés. 120 de los respondientes son femeninos, 98 son masculinos; todas ellos entre los 17 y los 19 años de edad. 


\subsection{Muestra}

Se inscribieron 897 alumnos de nuevo ingreso a la universidad. A todos ellos se les envió una encuesta. Se recibieron 291 respuestas, de las cuales se tienen datos completos (datos de identificación, nivel de ubicación) de 218.

\subsection{Instrumentos}

El examen de ubicación utilizado es el WebCAPE, creado por Perpetual Works. Es un examen que evalúa el dominio de una lengua a través de preguntas de opción múltiple. Existen versiones para varios idiomas. En el caso de este estudio, se utilizó la versión gramática de inglés como segunda lengua. El examen se presenta vía internet y es adaptivo; es decir, varían las preguntas de acuerdo a las respuestas, según un algoritmo. El examen de inglés como segunda lengua ha sido calibrado de acuerdo con los estándares del Consejo Norteamericano para la Enseñanza de Lenguas Extranjeras (ACTFL) y se adecúa al programa individual de cada institución que utiliza el examen.

La encuesta que se envió a los participantes fue de elaboración propia. Se incluyeron los factores que, según la literatura sobre el tema, tienen mayor impacto sobre el aprendizaje del idioma, como son, el tiempo de estudio, el estudiar en un país en donde se habla el idioma, la motivación para el estudio, entre otros. La encuesta se elaboró con Google Forms y se envió a los participantes a través del correo institucional.

Los alumnos de nuevo ingreso a la universidad presentan exámenes de ubicación de inglés en la semana próxima anterior al inicio de su primer semestre. Los exámenes son programados por cada escuela o facultad. Los alumnos tardan entre cinco y veinte minutos en responder.

La universidad maneja ocho niveles distintos. La tabla 1 muestra cada nivel con su equivalencia de acuerdo al Marco Común Europeo de Referencia (MCER).

Tabla 1. Niveles ofrecido por la institución y su equivalente según MCER

\begin{tabular}{|l|c|}
\hline \multicolumn{1}{|c|}{ Nivel institucional } & MCER \\
\hline Básico 1 & A1 \\
\hline Básico 2 & A2 \\
\hline Intermedio 1 & B1 \\
\hline Intermedio 2 & B2 \\
\hline Avanzado 1 & B2.2 \\
\hline Avanzado 2 & C1 \\
\hline Upper Advanced (Superior) & C1.2 \\
\hline
\end{tabular}


El contexto social y socio-económico en el cual se desenvuelve la mayoría de los participantes coadyuva a que tiendan a quedar ubicados en los niveles más bien altos. Los 218 participantes quedaron ubicados de la siguiente manera:

Tabla 2. Niveles de ubicación de los participantes

\begin{tabular}{|l|c|c|}
\hline \multicolumn{1}{|c|}{ Nivel de ubicación } & Número de alumnos & Porcentaje \\
\hline Básico 1 & 7 & 3.21 \\
\hline Básico 2 & 14 & 6.42 \\
\hline Intermedio 1 & 18 & 8.26 \\
\hline Intermedio 2 & 60 & 27.52 \\
\hline Avanzado 1 & 70 & 32.11 \\
\hline Avanzado 2 & 31 & 14.22 \\
\hline Upper Advanced (Superior) & 18 & 8.26 \\
\hline Total & 218 & 100 \\
\hline
\end{tabular}

\subsection{Procedimiento}

El procedimiento estadístico consiste en dos técnicas: regresión lineal múltiple y análisis de varianza (ANOVA) de un factor. En primer instancia se busca medir la posible influencia de las variable independientes: $X 1 \ldots X 11$ sobre la variable dependiente (Y1). Posteriormente se lleva a cabo el procedimiento ANOVA para contrastar si existe diferencia de medias en las variables poblacionales $X 1$ a $X 11$ y como variable factor $Y 1$. De ahí que a continuación se señalan algunas consideraciones teóricas para cada procedimiento (Hair et al, 1999; Triola, 2006;).

\subsubsection{Regresión lineal simple y múltiple para el análisis}

Teóricamente si la recta de regresión está dada por:

$$
y=\beta_{o}+\beta_{1} x
$$

Entonces cada valor observado de $Y_{i}$ para $x_{i}$ se entiende como el valor que se espera de $Y$ dado $x_{i}$ más el error, es decir:

$$
y_{i}=\beta_{o}+\beta_{1} x_{1 i}+e_{i}
$$

Ahora bien, si buscamos explicar el nivel de puntaje obtenido por el estudiante con una sola variable predictora, resulta muy complicado explicarlo de manera satisfactoria, ya que las causas asociadas a esa obtención de puntos del nivel de inglés, está asociada a varios aspectos, como ha quedado señalado en el punto 3.4, de ahí que, desde la teoría se establece que el comportamiento de las variables dependientes $Y$ puede ser explicada por $p$ variables predictoras $X_{1} \ldots X_{p i}$, a partir 
de $n$ observaciones, por lo que se sigue el modelo de regresión múltiple (Triola, 2006):

$$
y_{i}=\beta_{o}+\beta_{1} x_{1 i}+\beta_{2} x_{2 i}+\beta_{p} x_{p i} \ldots+e_{i}
$$

Si partimos de $i=1,2, \ldots n$ entonces lo podemos convertir en una serie de ecuaciones lineales a partir del siguiente modelo de regresión

$$
\begin{aligned}
& y_{1}=\beta_{o}+\beta_{1} x_{11}+\beta_{2} x_{21}+\beta_{p} x_{p 1} \ldots+e_{1} \\
& y_{2}=\beta_{o}+\beta_{1} x_{12}+\beta_{2} x_{22}+\beta_{p} x_{p 2} \ldots+e_{2} \\
& \ldots \ldots \ldots \ldots \ldots \ldots \ldots \ldots \ldots \ldots \ldots \ldots \ldots \ldots \ldots \ldots \ldots \ldots \ldots \ldots \ldots \ldots \ldots \ldots \ldots \ldots \ldots \ldots \ldots \ldots \ldots \ldots \\
& y_{n}=\beta_{o}+\beta_{1} x_{1 n}+\beta_{2} x_{2 n}+\beta_{p} x_{p n} \ldots+e_{n}
\end{aligned}
$$

En la forma matricial tenemos

$$
\left[\begin{array}{l}
y_{1} \\
y_{2} \\
\cdot \\
\cdot \\
\cdot \\
y_{n}
\end{array}\right]=\left[\begin{array}{l}
1 x_{11} x_{12} \ldots x 1_{p} \\
1 x_{21} x_{22} \ldots x 2_{p} \\
\ldots \ldots \ldots \ldots \ldots \ldots \ldots \\
\ldots \ldots \ldots \ldots \ldots \ldots \ldots \ldots \ldots \ldots \\
\ldots \ldots \ldots \ldots \ldots \ldots \\
1 x_{n 1} x_{n 2} \ldots x_{n p}
\end{array}\right]\left[\begin{array}{l}
\beta_{o} \\
\beta_{1} \\
\cdot \\
\cdot \\
\beta_{p}
\end{array}\right]+\left[\begin{array}{l}
e_{1} \\
e_{2} \\
\cdot \\
\cdot \\
\cdot \\
e_{n}
\end{array}\right]
$$

\subsubsection{ANOVA}

Para contrastar la hipótesis nula (Ho) de las medias poblacionales del puntaje obtenido en los niveles: básico 1 y 2, intermedio 1 y 2 , avanzado 1 y 2 y Upper Advanced $(\mathrm{K}>2)$ de que todas son iguales, versus la hipótesis alternativa ( $\mathrm{Ha})$ de que al menos uno de los puntajes obtenidos difiere con respecto al valor esperado.

$$
\begin{aligned}
& \text { Ho: } \mu_{1}=\mu_{2}=\mu_{1} \\
& H a: \ni \mu_{\iota} \neq \mu \ldots j=1,2 \ldots \ldots ., K
\end{aligned}
$$

De acuerdo a los fundamentos teóricos, para realizar el cálculo de ANOVA se requiere que se cumpla con los supuestos de:

- Las poblaciones (distribuciones de probabilidad de la variable dependiente correspondiente a cada factor) son normales. 
- Las $\mathrm{K}$ muestras sobre las que se aplican los tratamientos son independientes.

- Las poblaciones tienen todas igual varianza (homoscedasticidad).

El ANOVA se basa en la descomposición de la variación total de los datos con respecto a la media global (SCT), que bajo el supuesto de que $\mathrm{H}_{0}$ es cierta es una estimación de $\mathrm{S}^{2}$ obtenida a partir de toda la información muestral, en dos partes:

- Variación dentro de las muestras (SCD) o Intra-grupos, cuantifica la dispersión de los valores de cada muestra con respecto a sus correspondientes medias.

- Variación entre muestras (SCE) o Inter-grupos, cuantifica la dispersión de las medias de las muestras con respecto a la media global.

Dentro del procedimiento ANOVA, intervienen los siguientes elementos

Variación Total: $S C T=\sum_{J=1}^{K} \sum_{i=1}^{n j}\left(x_{i j}=\bar{X}\right)^{2}$

Variación Intra-grupos: $S C D=\sum_{J=1}^{K} \sum_{i=1}^{n j}\left(x_{i j}-\bar{X}_{j}\right)^{2}$

Media Global: $\bar{X}=\frac{\sum_{J=1}^{K} \sum_{i=1}^{n j} x_{i j}}{n}$.

Variación Inter-grupos: $S C E=\sum_{J=1}^{K}\left(\bar{X}_{j}-\bar{X}\right)^{2}{ }_{n j}$

Siendo xij el i-ésimo valor de la muestra j-ésima; $n j$ el tamaño de dicha muestra y $\bar{X}$ su media. Cuando la hipótesis nula es cierta SCE/K-1 y SCD/n-K son dos estimadores insesgados de la varianza poblacional y el cociente entre ambos se distribuye según una F de Snedecor con K-1 grados de libertad en el numerador y N-K grados de libertad en el denominador.

De ahí que, si $\mathrm{H}_{0}$ es cierta entonces se espera que el cociente entre ambas estimaciones sea aproximadamente igual a 1 , de forma que se rechazará $\mathrm{H}_{0}$ si dicho cociente difiere significativamente de 1 .

A continuación se exponen los resultados del modelo de Regresión múltiple $y$ el procedimiento de ANOVA para las diferencias de media en los puntajes básico 1 y 2 , intermedio 1 y 2 , avanzado 1 y 2 y Upper Advanced de las variables predictoras $X 1 \ldots X 12$. 


\section{Análisis de datos}

\section{A) Modelo de Regresión Múltiple}

Para el análisis de la regresión se forman dos grupos de variables; el primero lo conforman las variables: TIEMPOEST (X1), TIPOESCUELA (X2), ESTUDINLENGUAS (X3), ESTUDINMAPAR (X4), VIVIOFUERAPAIS (X5), RAZONPARAAPRENDER (X6), FRECULENTURA (X7) que conforman el grupo de antecedentes de estudio y las variables QUETALHABLAING (X8), QUETALESCRIBEING (X9), QUETALENTING (X10), QUETALLEESING (X11) conforman el grupo de percepción de habilidades, ambos grupos como variables predictoras que se confrontan con el puntaje obtenido como variable $Y 1$.

Figura 1. Modelo de regresión por grupos de variables predictoras (fuente propia)

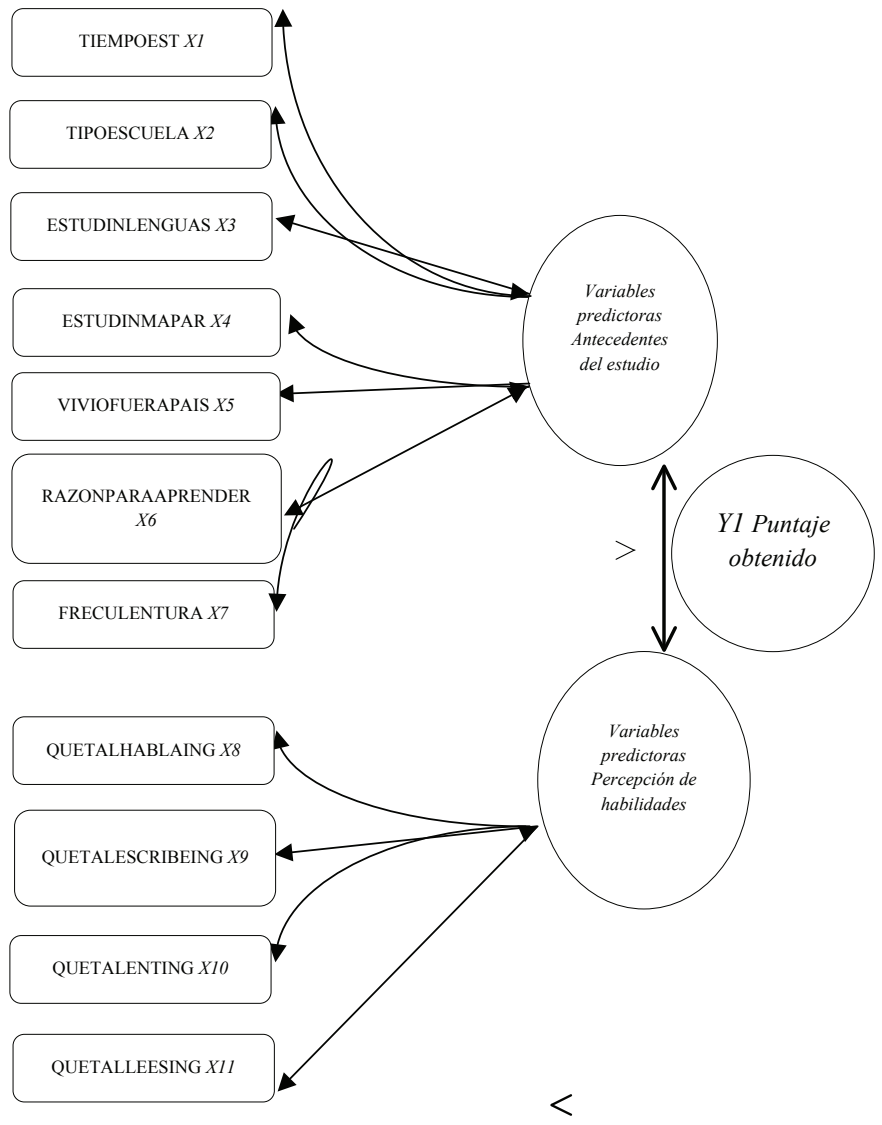


Los resultados de las variables predictoras sobre los antecedentes académicos muestran un valor $\mathrm{R}^{2}(0,324)$ revela que las variables del modelo de regresión contribuyen con un $32.4 \%$ al puntaje del examen. Además el coeficiente de correlación (0.569) indica una asociación aceptable entre las variables. El valor del estadístico (14.394) de prueba tiene un valor mayor que el valor en $\mathrm{F}$ en tablas, además, el valor de significancia es menor de 0.05; esto indica que las variables explicativas intervienen sobre la variable dependiente. La ecuación 1 expone las variables que define el modelo de regresión, el cual está constituido por tres variables: tiempo de estudio, tipo de escuela y si ha vivido fuera del país. El modelo muestra que el puntaje que el alumno obtiene en el examen depende de estas variables.

$Y=1.037+.213 T I E M P O E S T+.813 T I P O E S C U E L A+.280 V I V I O F U E R A P A I S+.618 \mathrm{FRECULENTURA} \mathrm{[ec1]} \mathrm{.}$

Resultados para el grupo de variables predictoras sobre la percepción de habilidades en inglés.

La tabla 6 muestra los estadísticos de la regresión, el valor del coeficiente $\mathrm{R}^{2}$ $(0,324)$ revela que la ecuación de regresión explica un $46.8 \%$ los valores observados El valor del coeficiente de correlación (0.684) indica una asociación aceptable entre las variables. Por otro lado, el valor del estadístico (46.911) de prueba tiene un valor mayor que el valor en $\mathrm{F}$ en tablas, además el valor de significancia es menor de 0.05 esto indica que las variables explicativas influyen de forma conjunta y lineal sobre la variable dependiente. La ecuación 2 expone las variables que define el modelo de regresión, el cual está constituido únicamente por dos variables: que tal escribe y que tal lee. El modelo indica que el puntaje que el alumno obtiene en el examen depende de que tal escribe y que tal lee el inglés.

\section{$Y=1.515+.526 Q U E T A L E$ RIBEING+.398UETALLEESING [ec.2]}

Los resultados de aplicar la técnica ANOVA indican que los alumnos que han dedicado el tiempo de estudio de la materia (TIEMPOEST), así como el tipo de escuela de donde proceden (TIEMPOEST) y si han estudiado lenguas (ESTUDINLENGUAS) además la frecuencia de lectura (FRECULENTURA) son variables que aumentan su puntaje en el examen ya que sus valores son menores de 0.05. Por el contrario si el alumno ESTUDINMAPAR O no tiene una razón para aprender RAZONPARAAPRENDER el puntaje en su examen no se ve afectado. También los resultados indican que el puntaje que el alumno obtiene en el examen varían de acuerdo a si habla, si escribe o si lee inglés. 


\section{Discusión de los resultados}

De acuerdo con las pruebas corridas, son tres variables dentro del grupo de antecedentes de estudio las que mayor impacto tienen sobre el nivel de ubicación.

La primera de ellas, tiempo de estudio, concuerda con lo que indica la literatura: el tiempo de estudio, es decir, las horas de instrucción recibidas en el idioma extranjero, lleva a un mayor dominio de la lengua (Brown, 2000; Lightbown $\&$ Spada, 2013; Muñoz, 2006), independientemente de la edad en la que se empezó a estudiar.

La segunda variable significativa - el tipo de escuela - también concuerda con otros estudios realizados en América Latina (Davies, 2009; Mejía, 2016; Rodríguez- Garcés, 2013), en donde los colegios particulares tienden a tener mejor nivel educativo que las escuelas públicas. Esto posiblemente se deba a la diferencia en el número de alumnos por aula en las instituciones públicas y privadas, mucho mayor en las primeras.

Una última variable, la de frecuencia con la que lees el inglés, también tiene una fuerte influencia sobre el dominio del idioma, de acuerdo a los exámenes de ubicación. Esta no aparece en la literatura consultada, aunque Krashen (2004) enfatiza del poder de la lectura placentera para el aprendizaje de una segunda lengua. Esta variable merece ser investigada con mayor detenimiento.

Por otra parte, en cuanto a las variables del rubro de percepciones, se ve que la percepción que tienen los respondientes de su propio dominio del idioma está alineado con la realidad, pues creer que se habla bien, creer que se lee bien, creer que se escribe bien y creer que se entiende bien son todas variables significativas.

\section{Conclusiones}

Existen dos creencias importantes en cuanto al aprendizaje de un segundo idioma. La primera de ellas es que, cuanto más pronto se empieza a aprender, será mejor. La segunda de estas creencias es que para aprender bien el idioma, no hay como vivir en el país donde se habla. Los hallazgos del presente estudio desmienten ambas creencias, pues se ve que ninguna de las dos es significativa. Sin embargo, se confirma la importancia del número de horas dedicadas al aprendizaje.

Los hallazgos parecen señalar que, más que una política que busque empezar la enseñanza del idioma lo más pronto posible, los esfuerzos deben encaminarse a incrementar el número de horas dedicadas al idioma, así como a fortalecer los programas de lectura en inglés. Ambas acciones llevarán a mejorar el dominio del idioma entre los aprendices. 


\section{Referencias bibliográficas}

Ardasheva, Y., \& Tretter, T. R. (2013). «Contributions of individual differences and contextual variables to reading achievement of English language learners: An empirical investigation using hierarchical linear modeling». TESOL Quarterly, 47(2), 323-351.

Baker-Smemoe, W; Dewey, D. P; Bown, J., \& Martinsen, R. A. (2014). «Variables affecting L2 gains during study abroad». Foreign Language Annals, 47(3), 464-486.

Brown, H.D. (2000). Principles of language teaching and learning, $4^{\text {th }}$ edition. White Plains, USA: Addison-Wesley Longman.

Davies, P. (2009) • «Strategic management of ELT in public educational systems: Trying to reduce failure, increase success». The Electronic Journal for English as a Second Language, 13(3), 1-22.

Fromkin, V.; Rodman, R., \& Hyams, N. (2011). An introduction to language, $9^{\text {th }}$ edition. Boston: Cengage Learning.

Graddol, D. (2006). English next. London: British Council.

Hair, J. F; Anderson, R. E.; Tatham, R. L. \& Black, W. C. (1999). Multivariate data analysis, $5^{\text {th }}$ edition. Spain: Prentice Hall.

Horwitz, E.K. (1988). "The beliefs about language learning of beginning university foreign language students». Modern Language Journal, 72, 283 294.

Krashen, S. D. (2004). The Power of Reading: Insights from the Research: Insights from the Research. ABC-CLIO.

Lightbown, P.M. \& Spada, N. (2013). How languages are learned, $4^{\text {th }}$ edition. Oxford: Oxford University Press.

López Montero, R.; Quesada Chaves, M. J.; Salas Alvarado, J. (s/f) «Factores socioculturales en el aprendizaje de un segundo idioma: el caso de la sede del Pacífico de la Universidad de Costa Rica». <http://srp.ucr.ac.cr/ sites/default/files/Factores\%20sociales\%20en\%20el\%20aprendizaje\%20 de\%20un\%20segundo\%20idioma.pdf $>$.

Mejía-Mejía, S. (2016). « Vamos hacia una Colombia bilingüe? Análisis de la brecha académica entre el sector público y privado en la educación del inglés.» Educ. Educ., 19(2), 223-237.

Mitchell, R., \& Myles, F. (2004). Second language learning theories, $2^{\text {nd }}$ edition. London: Hodder Arnold.

Muñoz, C. (ed.) (2006). Age and rate of foreign language learning. Clevedon, UK: Multilingual Matters. 
O’Donoghue, J. \& Calderón Martín del Campo, D. (Eds.) (2015). Sorry* El aprendizaje del inglés en México. Ciudad de México: Mexicanos Primero.

Rodríguez Garcés, C. (2015). «Competencias comunicativas en idioma inglés: La influencia de la gestión escolar y del nivel socioeconómico en el nivel de logro educativo en L2-inglés». Perfiles educativos, vol. 37, no. 149 jul./sep., México. Recuperado de: <http://www.revistas.unam.mx/index.php/perfiles/article/view/53123/47255>.

Sánchez, M.E., \& Diez, I. (2014). «La enseñanza del inglés como política pública de América Latina: Estudio comparado». Recuperado deः <http:// ifie.edu.mx/wp-content/uploads/2015/02/4.-Ingles-como-politica-publica -cuadro-comparativo.pdf>.

Santana, J.C. (2005) Moving towards metacognition. Proceedings of the Second International Online Conference on Second and Foreign Language Teaching and Research. Singhal, M. and Liontas, J. (eds). USA: The Reading Matrix Inc.

Swain, M. (1985). «Communicative competence: Some roles of comprehensible input and comprehensible output in its development». Input in second language acquisition, 15, 165-179.

Swain, M., \& Lapkin, S. (1995). «Problems in output and the cognitive processes they generate: A step towards second language learning»+Applied linguistics, 16(3), 371-391.

SEP. (2007). Programa sectorial de educación. Recuperado de <www.oei.es/historico/quipu/mexico/programa_sectorial_educacion_mexico.pdf $>$.

Triola, M. (2006). Estadística. Novena edición. México: Pearson Addison Wesley.

Valero Molina, N., \& Jiménez-Fernández, G. (2015). «Estudio exploratorio sobre dificultades en el aprendizaje de una segunda lengua: La opinión del profesorado». Espiral. Cuadernos del profesorado, 8(16), 1. 